\title{
Mapping Luminosity-Redshift Relationship to LTB Cosmology
}

\author{
Daniel J. H. Chung and Antonio Enea Romano \\ Department of Physics, University of Wisconsin, Madison, WI 53706, USA
}

\begin{abstract}
We derive a direct general map from the luminosity distance $D_{L}(z)$ to the inhomogeneous matter distribution $M(r)$ in the Lemaitre-Tolman-Bondi (LTB) cosmology and compute several examples. One of our examples explicitly demonstrates that it is possible to tune the LTB cosmological solution to approximately reproduce the luminosity distance curve of a flat FRW universe with a cosmological constant. We also discuss how smooth matter distributions can evolve into naked singularities due to shell crossing when the inhomogeneous "curvature" $E(r)$ is a function which changes sign.
\end{abstract}

\section{INTRODUCTION}

Standard Friedmann-Robertson-Walker (FRW) cosmology is characterized by the following features: homogeneity and isotropy when averaged on $\mathcal{O}(100)$ Mpc length scales, negligible spatial curvature, and a stress tensor which today has a chemical composition of approximately $70 \%$ dark energy and $30 \%$ pressureless dust. The standard inflationary embedding of FRW cosmology currently provides a successful picture of the universe. However, since the invocation of dark energy leads to a new coincidence problem, which is that dark energy dominance roughly coincides with the epoch of nonlinear structure formation, and since we still do not understand the cosmological constant problem, there has been a renewed interest in exploring alternative inhomogeneous cosmological models (which are not perturbatively related to FRW cosmologies) to see whether they might offer a competitively plausible picture of the universe [1, 2, 3, 4, 5, 6, 7, 8, 9, 10, 11].

Supernova luminosity distance measurements as a function of redshift offer compelling evidence for dark energy when interpreted in the context of FRW cosmologies [12, 13]. To interpret such data in the context of inhomogeneous cosmologies, it is often not particularly useful to average the underlying inhomogeneous variables to obtain a forced interpretation in terms of FRW cosmology. This is because generically there is no preferred spatial slicing to compute averages and no meaningful map between the physical observables and the time derivatives of the spatially averaged variables. In some sense, the observables contain more information than that which could be carried by averaged variables, and hence determining the appropriate smearing map on the observables which matches the information that could be carried by averaged variables requires a knowledge of the underlying inhomogeneities in the absence of special symmetries. Therefore, to characterize inhomogeneous cosmologies, it is useful to compute observables such as the luminosity distance function directly in terms of variables describing the underlying inhomogeneous geometry.

The Lemaitre-Tolman-Bondi (LTB) solution corresponds to a spherically symmetric exact solution to the Einstein equations with pressureless ideal fluid. Extensive analyses have been carried out for this model because it allows for investigations of inhomogeneities that cannot be represented as perturbative deviations from FRW cosmologies. The LTB solution is fixed by choosing three smooth functions $\left\{E(r), M(r), R_{0}(r)\right\}$, which allow an infinite number of different radial inhomogeneities. Most of the previous attempts to compute a cosmologically plausible luminosity distance $D_{L}(z)$ as a function of redshift $z$ consisted of finding a map from $\left\{E(r), M(r), R_{0}(r)\right\}$ to $D_{L}(z)$ or a Taylor expansion of $D_{L}(z)$ about $z=0$ [4, 9, 14, 15].

In this paper, we derive a map from $\left\{E(r), D_{L}(z), R_{0}(r)\right\}$ to $M(r)$. This has the advantage that the observed luminosity distance function $D_{L}(z)$ more directly dictates the underlying cosmological model, as opposed to having to guess the right $\left\{E(r), M(r), R_{0}(r)\right\}$ to produce the desired $D_{L}(z)$. In [5], a similar inverse problem (with a different choice of variables) was considered which focused on the situation with $E=0$, while in this paper, we will keep $E$ general. In using this new map, we find the interesting fact that the luminosity distance function is typically (depending on the choice of $E(r)$ ) an effective probe of the LTB geometry only for $z \lesssim 1$, since the luminosity distance function has a universal behavior in the limit that $\left\{R_{0}=0, E \rightarrow 0\right\}$. More precisely, $D_{L}(z)$ is fixed independently of $M(r)$ for $\left\{R_{0}=0, E=0\right\}$, since that limit corresponds to the $\Omega_{M}=1$ FRW universe. A negative consequence of this feature is that the differential equation map from $\left\{E(r), D_{L}(z), R_{0}(r)\right\}$ to $M(r)$ fails to be a numerically accurate map beyond $z \sim 1$. Nonetheless, we show how the numerical solution can be patched to a semi-analytic solution beyond $z=1$ to obtain a good fit (to within around $5 \%$ for the redshifts of interest) to obtain an LTB cosmology which reproduces the luminosity distance function of an FRW cosmology with $\Omega_{\Lambda}=0.7$ and $\Omega_{M}=0.3$.

Furthermore, we make a subsidiary observation about the class of LTB solutions which can mimic the observed $D_{L}(z)$. We find that if the radial inhomogeneity profile goes from $E(r)>0$ to $E(r)<0$ as $r$ increases while $M^{\prime}(r)$ is positive in that region, there is generically a danger of forming naked singularities, which can be interpreted as due to shell crossing. 
The order of presentation will be as follows. In the next section, we review the conventional approach to obtaining the luminosity distance as a function of redshift in the LTB cosmologies. In Sec. IIII we construct a set of differential equations (which we will refer to as the inversion method) which can be used to map the luminosity distance function into a particular LTB geometry. Afterwards, we apply the method to several examples. In Sec. $\mathbf{V}$ we discuss how smooth geometries can evolve into naked singularities when $E(r)$ switches signs. We then summarize and conclude. For completeness, we present the FRW luminosity distance with $\Omega_{M}+\Omega_{\Lambda}=1$ in Appendix A. In Appendix B, we write the LTB solution explicitly in a form that is not commonly found in the literature.

\section{CONVENTIONAL APPROACH}

The spherically symmetric Lemaitre-Tolman-Bondi (LTB) metric

$$
d s^{2}=d t^{2}-\frac{\left(R_{, r}\right)^{2}}{1+2 E(r)} d r^{2}-R^{2} d \Omega_{2}
$$

satisfies the Einstein equation with

$$
T_{\nu}^{\mu}=\operatorname{Diagonal}\left[\rho=\frac{M_{p l}^{2}}{4 \pi} \frac{M^{\prime}(r)}{R^{2} R_{, r}},-P=0,-P=0,-P=0\right]
$$

for differentiable functions $E(r)$ and $M(r)$ if $R(t, r)$ satisfies the partial differential equation

$$
\left(\frac{\partial_{t} R}{R}\right)^{2}=\frac{2 E(r)}{R^{2}}+\frac{2 M(r)}{R^{3}}
$$

The function $E(r)$ can be thought of as a generalized version of the spatial curvature parameter $\left(E(r) \propto-k r^{2}\right.$ in FRW), while $M(r)$ can be thought of as a generalized version of mass (for matter domination in FRW $M(r) \propto$ $\rho_{i} a_{i}^{3} r^{3} / M_{p l}^{2}$, where $\rho_{i}$ is an initial energy density, $a_{i}$ is an initial scale factor, and $r$ is the radial coordinate). The

function $R$ in the FRW limit takes the form $r a(t)$. The boundary condition for Eq. (3) is provided by the radial function $R_{0}(r) \equiv R\left(t_{0}, r\right)$, where $t_{0}$ is the time at which the boundary condition is set (note that this time is generically different from today).

The luminosity distance in LTB model is approximately given [9, 16] by

$$
\begin{aligned}
& D_{L}(z)=(1+z)^{2} R(t(z), r(z)) \\
& \frac{d r}{d z}=\frac{\sqrt{1+2 E(r(z))}}{(1+z) \partial_{t} \partial_{r} R(t(z), r(z))} \\
& \frac{d t}{d z}=\frac{-\left|\partial_{r} R(t(z), r(z))\right|}{(1+z) \partial_{t} \partial_{r} R(t(z), r(z))},
\end{aligned}
$$

where $t(z)$ and $r(z)$ physically represent the geodesic of the photon coming to us (located at $r=0$ ) starting from the radial distance of our horizon. Any photon we observe that starts from a closer radial distance will have a redshift which is the same as that experienced by the horizon photon when the ratio of the frequencies measured from any closer radial position is accounted for since redshift is independent of the frequency. Given an arbitrary choice of $M(r)$ and $E(r)$, Eqs. (5) and (6) are conventionally solved to obtain the luminosity distance function through Eq. (4). Angular diameter distance can be obtained from the luminosity distance function by dividing by $(1+z)^{2}$.

One can simplify Eqs. (5) and (6) in the regime in which $\partial_{t} R$ (i.e., the "local expansion" rate) maintains the same sign by rewriting Eq. (3) as

$$
\partial_{t} R=s \sqrt{2 E(r)+\frac{2 M(r)}{R(t, r)}},
$$


where $s \equiv \pm 1$ specifies whether there is local expansion or contraction. The solution to this differential equation requires a specification of a function of $r$ at the initial time hypersurface. We will define that function to be $R_{0}(r)$ : $R_{0}(r) \equiv R\left(t_{0}, r\right)$ (recall $t_{0}$ is not necessarily today). Hence, we compute

$$
\partial_{t} \partial_{r} R(t(z), r(z))=s \frac{E^{\prime}(r)+M^{\prime} / R-M \partial_{r} R / R^{2}}{\sqrt{2 E(r)+\frac{2 M}{R(t, r)}}},
$$

and rewrite Eqs. (5) and (6) as

$$
\begin{aligned}
& \frac{d r}{d z}=\frac{s \sqrt{1+2 E(r(z))} \sqrt{2 E(r)+\frac{2 M}{R(t, r)}}}{(1+z)\left[E^{\prime}(r)+M^{\prime} / R-M \partial_{r} R / R^{2}\right]} \\
& \frac{d t}{d z}=\frac{-s\left|\partial_{r} R(t(z), r(z))\right| \sqrt{2 E(r)+\frac{2 M}{R(t, r)}}}{(1+z)\left[E^{\prime}(r)+M^{\prime} / R-M \partial_{r} R / R^{2}\right]} .
\end{aligned}
$$

These have the advantage that there are no second derivatives appearing in the equations, but have the added assumption that the sign $s$ is a constant.

\section{INVERSION METHOD}

In Sec. III we explained the conventional approach of obtaining the luminosity distance function $D_{L}(z)$ for a given $\left\{M(r), E(r), R_{0}(r)\right\}$. In this section, we wish to stipulate $D_{L}(z)$ and solve for the class of $\left\{M(r), E(r), R_{0}(r)\right\}$ that corresponds to this luminosity distance. In particular, we will solve for $M(r)$ for a given $\left\{E(r), D_{L}(z), R_{0}(r)\right\}$. This inversion method has the advantage that the physical observable $D_{L}(z)$ can be mapped to the geometry of the underlying model directly without having to guess $M(r)$. The physics is simply that if one knows a single radial geodesic history of a photon which was emitted at an event $\left(t_{1}, r_{1}\right)$ and observed at $\left(t_{2}, r_{2}\right)$, one knows the full spacetime geometry in the region $\left(t_{1}<t<t_{2}, r_{1}<r<r_{2}\right)$ of the LTB solution owing to its spherical symmetry.

The basic equations for this goal also stem from Eqs. (9), (10), and the equation for luminosity distance, Eq. (4):

$$
R(z) \equiv R(t(z), r(z))=\frac{D_{L}(z)}{(1+z)^{2}} .
$$

Since Eqs. (9) and (10) depend on $\partial_{r} R$, we would like to find an expression for $\partial_{r} R$ as a function of $z$. To this end, we use the exact solution, which is given in Eq. B1]:

$$
\begin{gathered}
-\left(t-t_{0}\right) \sqrt{2} E(r)+\sqrt{R(t, r)} \sqrt{E(r) R(t, r)+M(r)}-Q(r)=\frac{M(r)}{\sqrt{E(r)}} \ln \left[\frac{\sqrt{R(t, r)}+\sqrt{\frac{M(r)}{E(r)}+R(t, r)}}{\sqrt{R_{0}(r)}+\sqrt{\frac{M(r)}{E(r)}+R_{0}(r)}}\right] \\
Q(r) \equiv \sqrt{R_{0}(r)} \sqrt{E(r) R_{0}(r)+M(r)}
\end{gathered}
$$

(or Eq. (B3) if $E<0$ ). Taking $\partial_{r}$ of Eq. (12), we obtain a linear equation for $\partial_{r} R$, which can be solved to find

$$
\begin{aligned}
\partial_{r} R= & \frac{f[R] \sqrt{R_{0}}}{f\left[R_{0}\right] \sqrt{R}} R_{0}^{\prime}(r)+\frac{E^{\prime}(r) f[R]}{2 E \sqrt{R}}\left(2 \sqrt{2}\left(t-t_{i}\right)+\frac{R_{0}^{3 / 2}}{f\left[R_{0}\right]}-\frac{R^{3 / 2}}{f[R]}-\frac{M_{1}}{E^{3 / 2}} \ln \frac{\sqrt{E R}+f[R]}{\sqrt{E R_{0}}+f\left[R_{0}\right]}\right. \\
& \left.+\frac{M_{1}^{2}}{E^{3 / 2}}\left[\frac{1}{M_{1}+E R_{0}+\sqrt{E R_{0}} f\left[R_{0}\right]}-\frac{1}{M_{1}+E R+\sqrt{E R} f[R]}\right]\right) \\
& +\frac{M^{\prime}(r)}{2 E}\left(-1+\frac{f[R] \sqrt{R_{0}}}{f\left[R_{0}\right] \sqrt{R}}+\frac{f[R]}{\sqrt{E R}}\left[2 \ln \frac{\sqrt{E R}+f[R]}{\sqrt{E R_{0}}+f\left[R_{0}\right]}+\frac{M_{1}}{f^{2}[R]+\sqrt{E R} f[R]}-\frac{M_{1}}{f^{2}\left[R_{0}\right]+\sqrt{E R_{0}} f\left[R_{0}\right]}\right]\right)
\end{aligned}
$$


where

$$
\begin{gathered}
f[X] \equiv \sqrt{M_{1}+E(r(z)) X} \\
M_{1}(z) \equiv M(r(z)) .
\end{gathered}
$$

Furthermore, the function $M^{\prime}(r)$ in Eqs. (9) and (10) can be replaced by

$$
M^{\prime}(r)=\frac{d M_{1}}{d z} / \frac{d r}{d z}
$$

Since there are three unknown functions $\left\{M_{1}(z), r(z), t(z)\right\}$, and Eqs. (9) and (10) (with appropriate substitutions for $\partial_{r} R$ and $M(r)$ ) provide only two independent equations, we require another independent equation. This is provided by $d R / d z$ through the chain rule:

$$
\frac{d}{d z} R=s \sqrt{2 E+\frac{2 M_{1}}{R}} \frac{d t}{d z}+\partial_{r} R \frac{d r}{d z}
$$

For a given set of $\left\{E(r), D_{L}(z), R_{0}(r)\right\}$, the set of differential equations Eq. (9), Eq. (10), and Eq. (18) can be solved for $\left\{t(z), r(z), M_{1}(z)\right\}$. Finally, to obtain $M(r)$, we invert $r(z)$ to obtain

$$
M(r)=M_{1}(z(r)) .
$$

In practice, as we discuss below, the procedure we just described is a bit more difficult because the differential equation can become singular for certain choices of $\left\{E(r), D_{L}(z), R_{0}(r)\right\}$. Also, for numerical implementation, it is useful to write Eqs. (9), (10), and (18) in the form

$$
\left(\begin{array}{c}
\frac{d t}{d z} \\
\frac{d r}{d z_{1}} \\
\frac{d x_{1}}{d z}
\end{array}\right)=(A)
$$

where $(A)$ is a matrix that does not contain any derivative terms. However, this matrix contains hundreds of terms consisting of combinations of $\left\{E, E^{\prime}, D_{L}, \frac{d}{d z} D_{L}, M_{1}, R_{0}\right\}$, and is not very illuminating in the general case.

Regarding the initial conditions, note that because the differential equation also generically has a $0 / 0$ division near $r=0$, a numerical implementation must typically set the boundary condition at a small but nonvanishing $r$. For this purpose, it is useful to linearize the system about $r=0$ to obtain intuition about the boundary condition near the origin. If we assume that $R(t, 0)=0, E(r=0)=0, \lim _{r \rightarrow 0} \frac{M}{R}=0$, and $R(t, r) \approx r \partial_{r} R(t, 0)$ near $r=0$, we find

$$
t-t_{i}=-\left.z \frac{d D_{L}}{d z}\right|_{z=0}+\mathcal{O}\left(z^{2}\right)
$$

where $t_{i}$ is the value of $t$ at $z=0$. Unfortunately, similar expressions for $M_{1}(z)$ and $r(z)$ near $z=0$ depend upon the assumption of the scaling behavior of $M(r)$ and $E(r)$ near $r=0$, and even the limiting expressions are algebraically complicated partly because of the presence of logs. For example, taking the ansatz $E(r) \propto r^{2}, r=r^{\prime}(0) z$, and $M_{1}(z)=M_{c} z^{3}$, we obtain from Eq. (20) a nonlinear consistency equation near $z=0$ for $\left\{r^{\prime}(0), M_{c}\right\}$. Hence, it is simpler to directly solve for the initial conditions numerically using Eq. (21), the ansatz $r=r^{\prime}(0) z$, and Eq. (20) in the limit $z \rightarrow 0$.

\section{EXAMPLES}

In this section, we will solve Eqs. (9), 10, and (18) for a variety of choices of $\left\{E(r), D_{L}(z), R_{0}(r)\right\}$ to demonstrate the inversion method described in Section $\amalg$ in physically relevant examples. 


\section{A. Flat FRW example}

As a first example of mapping $\left\{D_{L}(z), E(r)\right\}$ to $\{M(r), R(t, r)\}$, consider the matter dominated $(\Lambda=0)$ flat Friedmann-Robertson-Walker (FRW) universe

$$
\left\{R_{0}(r)=0, E(r)=0\right\}
$$

with $s=1$. The function $\partial_{r} R$ can be found from Eq. 14 as

$$
\partial_{r} R(t, r)=\frac{1}{3} \frac{M^{\prime}(r)}{M(r)} R(t, r) .
$$

The resulting differential equations (Eqs. (9), (10), and (18) are

$$
\begin{gathered}
\frac{d r}{d z}=\frac{\sqrt{\frac{2 M}{R(t, r)}}}{(1+z)\left[\frac{\frac{1}{d r} \frac{d}{d z}}{d z} M / R-\frac{1}{3} M^{\prime} / R\right]} \\
=\frac{\sqrt{2 M_{1} R}}{(1+z)\left[\frac{2}{3} \frac{d}{d z} M_{1}\right]} \frac{d r}{d z} \\
\frac{d t}{d z}=\frac{-R^{3 / 2}}{(1+z) \sqrt{2 M_{1}}} \\
\frac{d R}{d z}=\frac{1}{3} \frac{\frac{d}{d z} M_{1}}{M_{1}} R+\sqrt{\frac{2 M_{1}}{R}} \frac{d t}{d z} \\
=\left[1-\sqrt{\frac{2 M_{1}}{R}}\right] \frac{R}{3} \frac{d}{d z} \ln M_{1} .
\end{gathered}
$$

Note that the $d r / d z$ equation in this limit becomes independent of $d r / d z \neq 0$ because of the absence of $E$. Hence, this equation can be written as

$$
(1+z)\left[\frac{1}{3} \frac{d}{d z} \ln M_{1}\right]=\sqrt{\frac{R}{2 M_{1}}}
$$

Note that Eqs. 27] and 28) give

$$
R=c \frac{M_{1}^{1 / 3}}{1+z}
$$

where $c \equiv R(z=0) / M_{1}^{1 / 3}(z=0)$. Solving for $M_{1}$ and $R$, we find

$$
M_{1}=2^{3 / 2} c^{3 / 2}\left(1-\sqrt{\frac{1}{1+z}}\right)^{3}
$$

and

$$
R=\frac{\sqrt{2} c^{3 / 2}}{(1+z)}\left(1-\sqrt{\frac{1}{1+z}}\right) .
$$

Remarkably, $M(r(z))=M_{1}(z)$ is fixed without specifying $r(z)$. Note also that if we identify $\sqrt{2} c^{3 / 2}=\frac{2}{H_{0}}$, this $R(z)$ corresponds to the function implied by the luminosity distance of an $\Omega_{M}=1 \mathrm{FRW}$ universe. Indeed, inserting Eq. (23) into the expression for $T_{00}$ in Eq. (2), one finds that the stress tensor corresponds to a homogeneous FRW universe: $T_{00} \propto t^{-2}$ independently of $r$. Hence, this limit corresponds to the homogeneous matter density FRW limit. 
If we choose

$$
r(z) \propto\left(1-\frac{1}{\sqrt{1+z}}\right),
$$

which corresponds to the geodesic-redshift relationship in an FRW universe, we obtain

$$
M(r)=M_{1}(z(r)) \propto r^{3},
$$

which corresponds to the $M(r)$ leading to the familiar FRW solution. From Eq. [12, we can solve for $R$ finding $R \propto a r$. However, note that we can choose another $r(z)$ and obtain an infinite class of different $M(r)$ functions corresponding to the same luminosity distance function implied by Eq. (30).

We can turn this result around. As long as $M / R \gg E$, the luminosity distance curve no longer accurately probes the geometry of the LTB model since different geometries lead to approximately the same $R(z)=D_{L}(z) /(1+z)^{2}$. In particular, this means the inversion method will necessarily be unstable once the curvature term $E$ can be neglected. Schematically, we will have

$$
\frac{d r}{d z} \sim \frac{E R}{M_{1}} F+\frac{\sqrt{2 M_{1} R}}{(1+z)\left[\frac{2}{3} \frac{d}{d z} M_{1}\right]} \frac{d r}{d z},
$$

when $E R / M_{1}$ becomes small and $F \sim \mathcal{O}\left(\frac{d r}{d z}\right)$. Since $\frac{\sqrt{2 M_{1} R}}{(1+z)\left[\frac{2}{3} \frac{d}{d z} M_{1}\right]} \sim 1$ in the limit that $E R / M_{1} \rightarrow 0$, we have

$$
\frac{d r}{d z} \sim \frac{E R / M_{1}}{1-\frac{\sqrt{2 M_{1} R}}{(1+z)\left[\frac{2}{3} \frac{d}{d z} M_{1}\right]}} F \sim \frac{0}{0},
$$

which creates an unstable differential equation for $\frac{d r}{d z}$ in this limit.

\section{B. Void Model}

We will now reproduce the void model of [4] by applying the inversion method as a nontrivial check.

First, let us review the solution of [4]. Their ansatz for $E(r)$ and $M(r)$ can be written as

$$
\begin{gathered}
E(r)=\frac{1}{2} H_{\perp, 0}^{2} r^{2}\left(\beta_{0}-\frac{\Delta \beta}{2}\left[1-\tanh \frac{r-r_{0}}{2 \Delta r}\right]\right) \\
M(r)=\frac{1}{2} H_{\perp, 0}^{2} r^{3}\left(\alpha_{0}-\frac{\Delta \alpha}{2}\left[1-\tanh \frac{r-r_{0}}{2 \Delta r}\right]\right) \\
R\left(t_{r e c}, r\right)=a_{r e c} r,
\end{gathered}
$$

with

$$
\left\{\alpha_{0}=1, \beta_{0}=0, \Delta \beta=-\Delta \alpha=-0.9, \Delta r=0.4 r_{0}, r_{0} \approx \frac{1}{5 H_{0}}, H_{\perp, 0} \approx H_{0}\right\},
$$

where $H_{0} \approx 50 \mathrm{~km} / \mathrm{s} / \mathrm{Mpc}$ and $a_{r e c} \sim 10^{-3}$ is the effective scale factor at recombination. Note that in their solution, $E(r)>0$ never changes sign. Hence, the solution is always in a "locally open" universe (recall $E(r) \sim-k r^{2}$ in the FRW limit). The resulting luminosity distance $D_{L}^{\text {void }}(z)$ fits the supernovae luminosity distance well [4].

To use the inversion method to derive this $M(r)$, we set $E(r)$ to Eq. 35, $R(z)=D_{L}^{\operatorname{void}}(z) /(1+z)^{2}$, and $R_{0}(r)$ equal to Eq. (37) and solve the differential equations Eqs. (9), (10), and (18) subject to the boundary condition $\left\{t\left(z_{i}\right) \approx 0.855 \frac{1}{H_{0}}, r\left(z_{i}\right) \approx 0, M_{1}\left(z_{i}\right) \approx 0\right\}$, where $z_{i} \approx 0$ and $\lim _{r \rightarrow 0} M_{1}(z(r)) / r^{3} \approx 0.084 H_{0}^{2}$ was taken to match Eq. [38 [19]. These boundary conditions correspond to ray tracing starting from the "center of the universe" where observations are assumed to be made.

The results of the $r(z)$ and $M(r)$ reconstruction are shown in Fig. 11 The solid curve was constructed using the inversion method and should ideally match the dashed curve. However, the inversion method breaks down for $z \sim 0.6$ because there $E R / M \rightarrow 0$, in which case the $d r / d z$ equation becomes unstable as explained in SubsectionIVA To see this explicitly, we plot $E R / M$ as a function of redshift $z$ in Fig. 2 

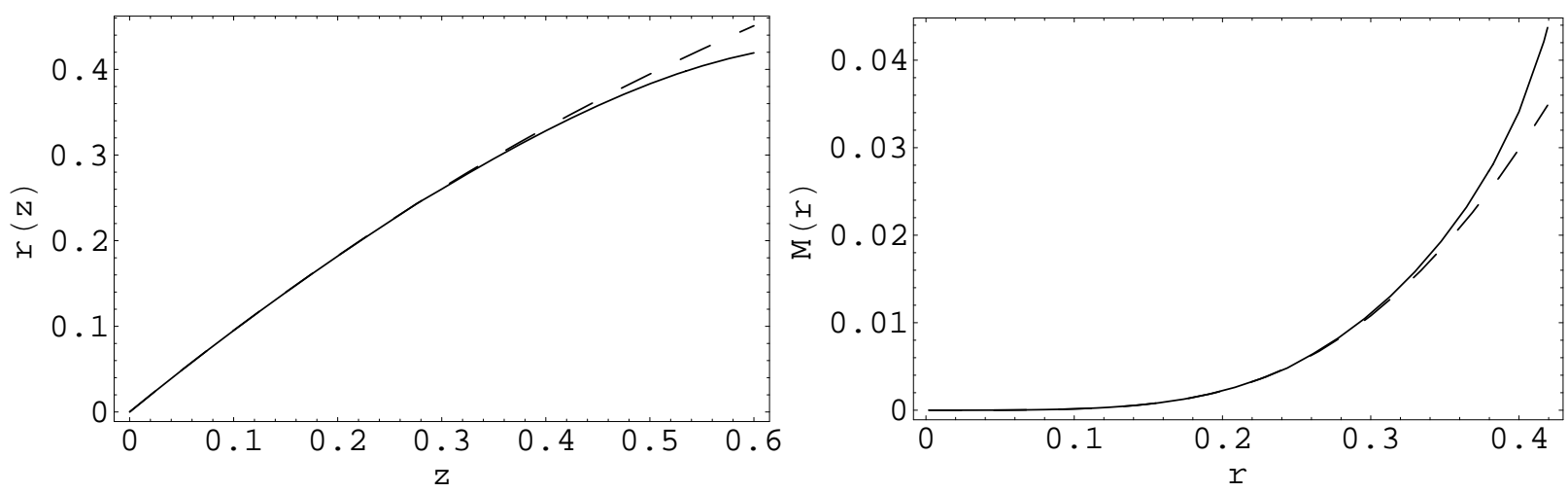

Figure 1: In the graph on the left, the solid curve shows the reconstructed $r(z)$ (in units of $1 / H_{0}$ ) through the inversion method and the dashed curve shows the $r(z)$ that would be obtained if $M(r)$ of Eq. 36 were given as an input. The breakdown of the inversion method for this model around $z \approx 0.6$ is apparent and is explained in the text. In the graph on the right, the solid curve gives the $M(r)$ as determined through the numerical evaluation of $M_{1}(z(r))$, and the dashed curve gives $M(r)$ as given by Eq. 36 .

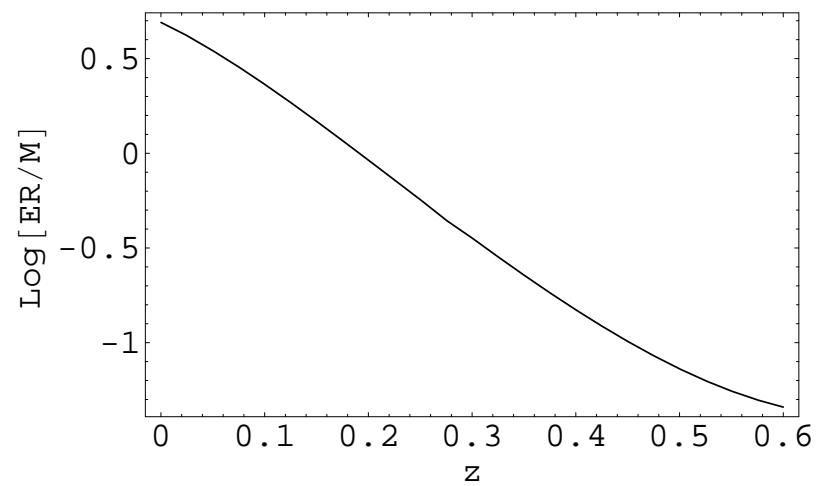

Figure 2: $E R / M$ versus photon redshift $z$. For $z=0.6, E R / M \sim 10^{-2}$ and the $d r / d z$ differential equation is unstable. Another way to view the instability is that the luminosity distance function is not sensitive to the geometry for $z \gtrsim 0.6$ in this model.

\section{Cosmological Constant without Cosmological Constant}

Until now, we have given two examples of using the inversion method to reproduce known LTB models. In this subsection, we construct an LTB model which reproduces an identical luminosity distance to that produced by an FRW universe with $\Omega_{M}=0.3$ and $\Omega_{\Lambda}=0.7$ up to a finite redshift. In particular, we will set $R(z)=D_{L}(z) /(1+z)^{2}$, with $D_{L}(z)$ given by Eq. A1.

Although the inversion method is unstable for $z \gtrsim z_{c}$, where $z_{c}$ is a cutoff redshift (whose generic existence is suggested by the two examples previously presented), we can use the inversion method for $z<z_{c}$ and then smoothly patch that model (i.e. the function $M(r)$ ) on to a function $M(r) \sim r^{3}$. Since the luminosity distance function is sensitive to the cosmological constant only for $z \lesssim 1$, such models tend to give good fits to the observed luminosity distance data.

For our trial example, in addition to setting $R(z)$ according to Eq. A1 and $R_{0}(r)=0$, we choose

$$
E(r)=\frac{1}{2} H_{0}^{2} r^{2} \exp \left[-2 H_{0} r\right]
$$

which has a local spatial curvature only for $r \approx 0$. The solution $t(z), r(z), M_{1}(z)$ is obtained by solving Eqs. 9], (10), and (18) with the initial conditions to the differential equations set as outlined near Eq. (21). Explicitly, we take $t\left(z_{i}\right)=0.93 / H_{0}, r\left(z_{i}\right)=0.97 z_{i} / H_{0}, M_{1}\left(z_{i}\right)=0.028 r^{3}\left(z_{i}\right)$, and $z_{i}=10^{-5}$. As previously discussed near Eq. (21), we solved linearized equations to obtain $t\left(z_{i}\right)$ and $r\left(z_{i}\right)$, assuming $M_{1}\left(z_{i}\right)=0.028 r^{3}\left(z_{i}\right)$. (The coefficient 0.28 in 


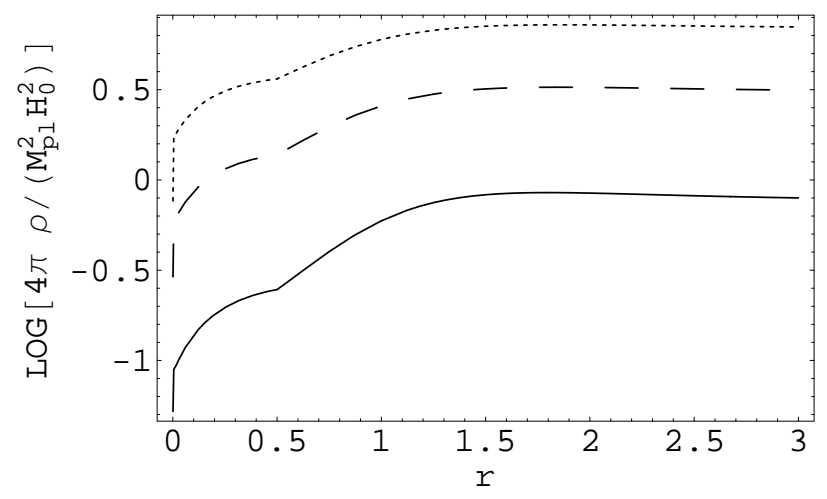

Figure 3: Using the inversion method, we have computed the energy density $\rho(t, r)$ corresponding to the luminosity distance of an FRW universe with $\Omega_{M}=0.3$ and $\Omega_{\Lambda}=0.7$. The solid, dashed, and dotted curves correspond to $\rho(t, r)$ at times $t_{\text {today }}, t_{\text {today }} / 2$, and $t_{\text {today }} / 3$, respectively. With $H_{0}=70 \mathrm{~km} / \mathrm{s} / \mathrm{Mpc}, t_{\text {today }}$ is 13 billion years. The redshift corresponding to $r \approx 0.5 / H_{0}$ is $z \approx 0.4$. The energy density at $r=0$ is nonzero: $4 \pi \rho\left(t_{\text {today }}, 0\right) /\left(M_{p l}^{2} H_{0}^{2}\right) \sim 10^{-3}$.

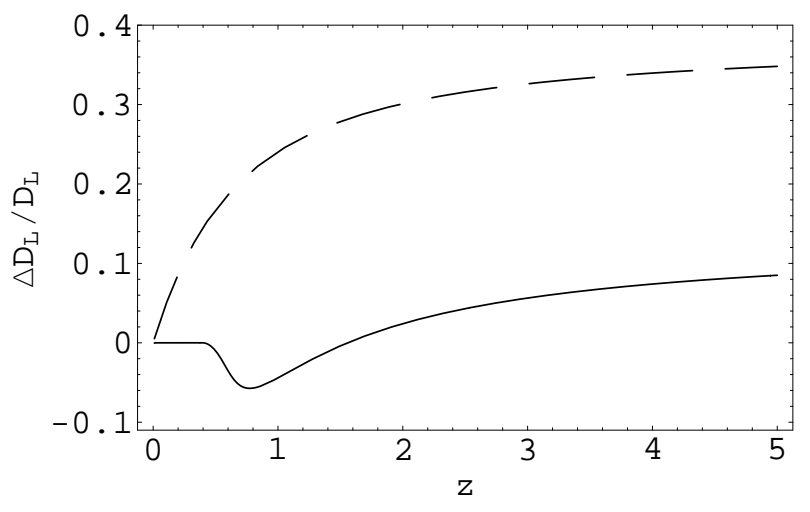

Figure 4: The solid curve corresponds to $\left(D_{L}^{\Lambda=0.7}(z)-D_{L}^{\operatorname{model}}(z)\right) / D_{L}^{\Lambda=0.7}(z)$ which corresponds to the deviation of the numerically constructed "model" luminosity distance curve from the FRW curve (where the FRW model is one with $\left\{\Omega_{\Lambda}=0.7, \Omega_{M}=\right.$ $0.3\}$ ). For comparison, we plot the dashed curve, which corresponds to $\left(D_{L}^{\Lambda=0.7}(z)-D_{L}^{\Lambda=0}(z)\right) / D_{L}^{\Lambda=0.7}(z)$, where $D_{L}^{\Lambda=0}$ corresponds to a flat matter-dominated FRW model without any cosmological constant. It is clear that the model reproduces the luminosity curve exactly from $z=0$ up to $z=0.4$ (by construction) and there is a less than about 5\% deviation from the FRW $\left\{\Omega_{\Lambda}=0.7, \Omega_{M}=0.3\right\}$ luminosity distance curve until about $z=3$. Furthermore, the deviation error is seen to plateau at large redshifts.

$M_{1}\left(z_{i}\right)$ is related to the energy density at $r=0$ through Eq. (2).) The differential equation was then solved from $z \approx 0$ to $z=z_{c} \approx 0.4$. From approximately this redshift onward [20], the inversion method is unstable because of the form $0 / 0$. (It is unclear from our analysis whether this instability is purely numerical or whether this instability can be removed by a reformulation of the equation and perturbations of the boundary conditions. We defer this question to a future work.) Hence, we fit a smooth $M(r)$ function starting from close to this point:

$$
M(r)=\left\{\begin{array}{cc}
M_{1}^{\text {num }}\left(z^{\text {num }}(r)\right) & r \leq 0.5 / H_{0} \\
C_{1}\left(r+C_{2}\right)^{3} & r>0.5 / H_{0}
\end{array}\right.
$$

where the superscript "num" refers to the numerical solutions to Eqs. (9), (10), and (18) and $C_{1}$ and $C_{2}$ are adjusted to match the value and the $r$ derivative at $r=0.5$. We then take the extended $M(r)$ and solve Eq. (B1) to obtain $R(t, r)$ numerically. Finally, we then solve Eqs. (4) and (5) to obtain the full luminosity distance beyond $z=z_{c} \approx 0.4$. Note that a functional choice of $C_{1}\left(r+C_{2}\right)^{3}$ was made to obtain an approximately homogeneous $\rho(t, r)$ for $r \sim 1 / H_{0}$.

The resulting solutions can be seen in Figs. 3 and 4 Clearly, the inhomogeneous model, whose energy density is shown in Fig [3] reproduces the luminosity distance of the $\left\{\Omega_{\Lambda}=0.7, \Omega_{M}=0.3\right\}$ FRW model exactly from $z=0$ to 
$z=0.4$ and the luminosity distance curve deviation from that of the FRW model is less than around $5 \%$ until $z=3$. (The $\left\{\Omega_{\Lambda}=0.7, \Omega_{M}=0.3\right\}$ FRW model is known to give a good fit to the supernova data.)

Hence, we have explicitly demonstrated that the LTB model can be tuned to obtain a luminosity-distance-redshift relationship which accurately reproduces that of a standard flat FRW universe with a cosmological constant and dark matter. This solution differs from previously proposed solutions in that the luminosity distance curve is exactly that of the FRW universe with a cosmological constant from $z=0$ to $z=0.4$. It is interesting to note that the energy density is approximately homogeneous for large $r$ but has a void close to $r=0$ (see Fig. 3], similarly to the model of [4].

\section{NAKED SINGULARITY FORMATION}

In this section, we demonstrate that the LTB model is susceptible to the formation of naked singularities when $E(r)$ switches sign from positive to negative as $r$ increases while $M^{\prime}(r)$ is positive in that region. More specifically, we consider situations in which $k(r) \sim-2 E(r) / r^{2}$ (i.e., the "local" spatial curvature factor) makes a smooth transition from an "underdense" universe to an "overdense" universe as $r$ crosses $r_{0}$ from below. This naked singularity may be interpreted as due to formation of caustics arising from matter accretion. Since any realistic system has nonzero pressure at sufficiently small length scales, this singularity is unphysical and is an artifact of pressureless dust approximation. This type of naked singularity would develop if one naively smooths out inhomogeneity profiles such as the one used by [2].

Now, note that because of the expression for the energy density in Eq. (2), for the energy density to be positive, $M^{\prime}(r)$ must have the same sign as $R, r$. Using Eq. (B5), which is valid for $E R / M \ll 1$ (which is almost always true in the vicinity of $r=r_{0}$, the point at which $E(r)$ changes sign smoothly), let us consider the situation in which $R_{0}(r)=0$ and $t_{0}=0$ (the case considered by [2]). We find

$$
R,_{r}=P(t, r)\left[E^{\prime}(r)-\frac{E(r)}{3} \frac{M^{\prime}(r)}{M(r)}+\frac{5 \cdot 2^{1 / 3}}{3 \cdot 3^{2 / 3}} \frac{M^{\prime}(r)}{t^{2 / 3} M^{1 / 3}(r)}\right]
$$

where

$$
P(t, r)=\frac{3 \cdot 3^{1 / 3}}{5 \cdot 2^{2 / 3}} \frac{t^{4 / 3}}{M^{1 / 3}(r)}
$$

Supposing that the last term proportional to $M^{\prime}(r) /\left(t^{2 / 3} M^{1 / 3}\right)$ can be neglected compared to the $E^{\prime}(r)$ term and $M^{\prime}(r)>0$, we have the condition

$$
E^{\prime}(r)>\frac{E(r)}{3} \frac{M^{\prime}(r)}{M(r)}
$$

if $M>0$. If we choose a smooth $E(r)$ which goes from a positive value to a negative value as $r$ increases past $r_{0}$, the Taylor expansion of $E(r)$ is then required to have the form

$$
E(r)=E_{1}\left(r_{0}\right)\left(r-r_{0}\right)+\frac{1}{2} E_{2}\left(r_{0}\right)\left(r-r_{0}\right)^{2}+\ldots
$$

with $E_{1}<0$, where $E_{n}$ corresponds to the $n$th derivative of $E(r)$. Hence, in a sufficiently small neighborhood of $r=r_{0}$, for $E_{1}\left(r_{0}\right) \neq 0$ we have the condition

$$
\frac{\left(r-r_{0}\right)}{3} \frac{M^{\prime}(r)}{M(r)}>1
$$

If we assume $M^{\prime} / M$ does not switch sign at $r=r_{0}$, this condition is clearly not satisfied in the vicinity of $r_{0}$. This means that when the $E^{\prime}(r)$ term dominates the $R, r$ expression, the energy density cannot remain positive definite.

Now, suppose that the $M^{\prime}(r) /\left(t^{2 / 3} M^{1 / 3}\right)$ term dominates over the $E^{\prime}$ term in Eq. [39], which would certainly be true near the big-bang singularity at $t \rightarrow 0$. We find the following condition for $M^{\prime}>0$ and $M>0$

$$
\frac{5 \cdot 2^{1 / 3}}{3^{2 / 3}} \frac{M^{2 / 3}(r)}{t^{2 / 3}}>E(r)
$$




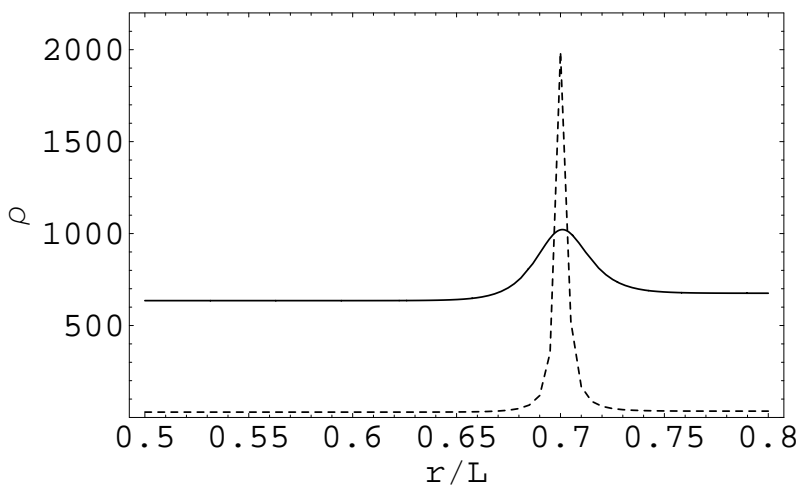

Figure 5: The energy density is plotted as a function of radius for times $t=0.01 L$ (solid) and $t=0.042 L$ (dotted) for the model of Eqs. 45 and (46) with $\left\{r_{0}=0.7 L, \kappa=50, \rho_{0}=3.5 \frac{1}{L^{2}}\right\}$. As expected, the energy density diverges as $R, r \rightarrow 0$ at $r \approx r_{0}$ near the time $t \gtrsim 0.04 L$. Note also that the energy density for $r>r_{0}$ is larger than $r<r_{0}$, since one side is overdense while the other side is underdense.

which can be satisfied in the vicinity of $r=r_{0}$. This means that energy can be positive definite in these regime. Hence, we arrive at a naively puzzling question why the energy density, which is initially positive definite near the big bang singularity, develops into a negative energy density when $E^{\prime}(r)$ term governs the value of $\rho$. The answer is that a naked singularity develops during the course of matter evolution.

To see this in a more obvious way, let us take a concrete model of

$$
M(r)=\rho_{0} \frac{a_{0}^{3} r^{3}}{6}
$$

and

$$
E(r)=\frac{-r^{2}}{2 L^{2}} \tanh \left(\kappa \frac{\left(r-r_{0}\right)}{L}\right),
$$

which represents a version of the model of [2] with the step function smoothed out. We can explicitly solve for the coordinate where $R,{ }_{r}$ becomes negative by solving $R,,_{r}=0$ :

$$
\frac{10}{3 \cdot 6^{1 / 3}} \frac{L^{2} a_{0}^{2} \rho_{0}^{2 / 3}}{t^{2 / 3}}-\frac{\kappa r}{L} \operatorname{sech}^{2}\left[\frac{\kappa\left(r-r_{0}\right)}{L}\right]-\tanh \left[\frac{\kappa\left(r-r_{0}\right)}{L}\right]=0
$$

Expanding about $r=r_{0}$ to quadratic order in $r-r_{0}$, we find the radius at which $R,,_{r}$ becomes zero to be

$$
\frac{r_{s}-r_{0}}{L}=\frac{L}{\kappa^{2} r_{0}} \pm \frac{1}{\kappa} \sqrt{\frac{-5 \cdot 6^{2 / 3}}{9 \kappa} \frac{L}{r_{0}}\left[a_{0}^{2} L^{2}\right] \rho_{0}^{2 / 3} t^{-2 / 3}+\frac{L^{2}}{\kappa^{2} r_{0}^{2}}+1}
$$

Requiring that the solution be real results in the condition

$$
t \gtrsim \sqrt{5}\left[\rho_{0} a_{0}^{3} L^{3}\right]\left(\frac{L}{\kappa r_{0}}\right)^{3 / 2}
$$

after which time, $R, r_{r}$ has a value of zero near $r \approx r_{0}+\frac{L^{2}}{\kappa^{2} r_{0}}$. Comparing this time with the time $t_{c}$ at which the overdense part of the universe starts to collapse $\left(t_{c} \approx \frac{\pi a_{0}^{3} L^{3} \rho_{0}}{6}\right)$, we see that if $\kappa \gg 1$ while $\mathcal{O}\left(r_{0}\right) \sim \mathcal{O}(L), R, r$ will reach zero before $t$ reaches $t_{c}$.

When $R, r$ reaches zero at nonzero $r=r_{s}$ without $M^{\prime}(r)$ vanishing, we have a Ricci curvature singularity there. We have also checked the divergence of the energy density near the spacetime region of the naked singularity for several numerical examples, one of which is shown in Fig. 5 Since no metric element became zero before the singularity comes into existence, we see that the curvature singularity is not protected by a horizon; i.e., it is a naked singularity. As is well known [17], naked singularities can develop with perfect fluid systems. Hence, if $E(r)$ changes sign at 


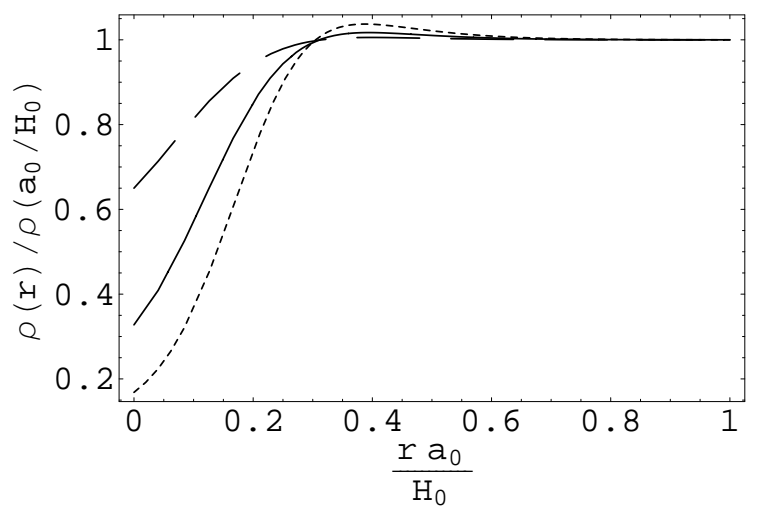

Figure 6: The energy density as a function of radius for three different times. The dotted curve corresponds to $t=0$ (recombination), the dashed curve corresponds to $t=0.1 / H_{0}$, and the solid curve corresponds to $t=0.5 / H_{0}$.

$r=r_{0}$ with $M(r)>0$ and $M^{\prime}(r)>0$ in the vicinity of $r_{0}$, a naked singularity develops, and the Einstein equations break down in that region. Furthermore, if we naively continue using the Einstein equations past the development of naked singularities, one finds a negative energy density region which is unphysical as expected.

Here we note that the model of [4] does not have any naked singularity problems associated with shell crossing. To see this, we will use the analytic approximation of Eq. (B5) applicable to the region in which $|E(r) R / M| \ll 1$. In the scenario specified by Eqs. (38), we have

$$
\left|\frac{E\left(r_{0}\right) R\left(t, r_{0}\right)}{M\left(r_{0}\right)}\right| \approx\left|0.3\left(10^{-4}+4 H_{0} t\right)^{2 / 3}\left(1-\frac{2 \times 10^{-8}}{10^{-4}+4 H_{0} t}+0.06\left[10^{-4}+4 H_{0} t\right]^{2 / 3}\right)\right| \ll 1
$$

until the time $t>1 / H_{0}$. One can then use Eq. (B5) in this regime to evaluate $R, r / r$ and find that the energy density does not diverge anywhere. The key reason is that $E(r)$ never switches sign, unlike the previous case.

There is a peculiar physical feature of the energy density as a function of time, as shown in Figure 6 Some of the matter density from the transition region near $r_{0}$ initially transfers to the $r=0$ region before being diluted away by curvature dominated expansion. In other words, the density near $r=0$ is not a monotonic function of time because the initial conditions are set up such that the fluid is flowing towards $r=0$.

\section{CONCLUSION}

In this paper, we have derived a set of differential equations in the context of LTB cosmologies (spherically symmetric pressureless dust solutions to the Einstein equations) which can be numerically solved to obtain almost any luminosity-distance-redshift relationship that can be produced by a homogeneous and isotropic FRW model. We have solved this set of differential equations for several examples to demonstrate the feasibility of our inversion method. Unlike many other methods in the literature, our method can be used to dial in the geometry that generates the desired luminosity distance exactly in a finite redshift interval. We have also given explicit examples of naked singularity formation in LTB cosmologies in the region where $E(r)$ changes sign.

Although our work serves as a step towards building cosmological models competitive to the standard FRW cosmology, the research program is far from completion. Toy models such as the LTB cosmologies are arguably not yet convincing contenders for compelling alternatives to standard inflationary cosmology, since being at the center of the universe requires giving up the Copernican principle (though recently there has been some effort to alleviate this problem [7]) and there is not yet a convincing structure formation history that could explain such radial inhomogeneities. Nonetheless, it is not obvious whether this class of LTB models can be ruled out from current observations [4]. Furthermore, there is some evidence that our galaxy is in a void, qualitatively similar to the voids presented in two of our numerical examples. However, the existence of the void is still currently being investigated (see for example [18]), and whether the magnitude of the void is sufficient to cause the redshift effects presented in this paper is unclear. Regarding tests of LTB cosmologies, any physical probe testing the isotropy of the universe from a point separated sufficiently far away from us could in principle be useful. We leave this problem for future investigation. 


\section{Acknowledgments}

We thank E. Kolb for discussions and suggesting this problem to us. DJHC also thanks D. McCammon for useful discussions and L. Everett for comments on the manuscript. The work of DJHC and AER was supported in part by the DOE Outstanding Junior Investigator Program through Grant No. DE-FG02-95ER40896 and NSF Grant No. PHY-0506002. AER was supported in part by the Wisconsin Alumni Research Foundation.

[1] J. W. Moffat, JCAP 0510, 012 (2005) |arXiv:astro-ph/0502110|.

[2] Y. Nambu and M. Tanimoto, arXiv:gr-qc/0507057

[3] A. Ishibashi and R. M. Wald, Class. Quant. Grav. 23, 235 (2006) |arXiv:gr-qc/0509108|.

[4] H. Alnes, M. Amarzguioui and O. Gron, Phys. Rev. D 73, 083519 (2006) |arXiv:astro-ph/0512006|.

[5] R. A. Vanderveld, E. E. Flanagan and I. Wasserman, Phys. Rev. D 74, 023506 (2006) |arXiv:astro-ph/0602476|.

[6] P. S. Apostolopoulos, N. Brouzakis, N. Tetradis and E. Tzavara, JCAP 0606, 009 (2006) |arXiv:astro-ph/0603234|.

[7] T. Kai, H. Kozaki, K. i. nakao, Y. Nambu and C. M. Yoo, arXiv:gr-qc/0605120

[8] J. W. Moffat, JCAP 0605, 001 (2006) |arXiv:astro-ph/0505326|.

[9] M. N. Celerier, Astron. Astrophys. 353, 63 (2000) |arXiv:astro-ph/9907206|.

[10] T. Biswas, R. Mansouri and A. Notari, arXiv:astro-ph/0606703

[11] C. H. Chuang, J. A. Gu and W. Y. Hwang, arXiv:astro-ph/0512651

[12] A. G. Riess et al. [Supernova Search Team Collaboration], Astron. J. 116, 1009 (1998) |arXiv:astro-ph/9805201|.

[13] S. Perlmutter et al. [Supernova Cosmology Project Collaboration], Astrophys. J. 517, 565 (1999) |arXiv:astro-ph/9812133|.

[14] N. Sugiura, K. i. Nakao and T. Harada, Phys. Rev. D 60, 103508 (1999) |arXiv:gr-qc/9911090|.

[15] N. Mustapha, B. A. Bassett, C. Hellaby and G. F. R. Ellis, Class. Quant. Grav. 15, 2363 (1998) |arXiv:gr-qc/9708043|.

[16] J. Kristian and R. K. Sachs, Astrophys. J. 143, 379 (1966).

[17] R. M. Wald, General Relativity (Univ. Chicago Press, Chicago, IL 1984).

[18] W. J. Frith, N. Metcalfe and T. Shanks, arXiv:astro-ph/0509875

[19] The value of $t\left(z_{i}\right)$ is chosen for numerical convenience and is essentially arbitrary since it merely corresponds to a shift of the origin of time.

[20] The differential equation does not become unstable until $z \approx 0.505$, but we stopped it earlier to obtain a smoother fit to the $M(r) \sim r^{3}$ ansatz.

\section{Appendix A: FRW LUMINOSITY DISTANCE}

The FRW luminosity distance in flat FRW universe with pressureless dust fraction $\Omega_{M}$ and cosmological constant fraction $\Omega_{\Lambda}$ without spatial curvature $\left(\Omega_{M}+\Omega_{\Lambda}=1\right)$ is given by

$$
\begin{gathered}
D_{L}(z)=\frac{1+z}{H_{0}} \int_{1}^{1+z} d y I(y) \\
I(y) \equiv \frac{1}{\sqrt{\Omega_{\Lambda}+\left(1-\Omega_{\Lambda}\right) y^{3}}}
\end{gathered}
$$

where the integral is numerically a value of order 1 with a logarithmic dependence on $z$. Note that a Taylor expansion is not very efficient approximation of the integral as fifth order expansion gives a good fit to only about $z \approx 1.5$.

To check the plausibility of this expression, consider the solvable case of matter dominated universe $\Omega_{\Lambda}=0$ :

$$
\begin{aligned}
D_{L}(z) & =\frac{1+z}{H_{0}} \int_{1}^{1+z} d y y^{-3 / 2} \\
& =2 \frac{1+z}{H_{0}}\left[1-\frac{1}{\sqrt{1+z}}\right]
\end{aligned}
$$

which is the familiar expression. If this is matched to LTB model, we would write

$$
\begin{aligned}
R(z) & =\frac{D_{L}(z)}{(1+z)^{2}} \\
& =2 \frac{1}{H_{0}}\left[\frac{1}{1+z}-\frac{1}{(1+z)^{3 / 2}}\right] .
\end{aligned}
$$




\section{Appendix B: EXPLICIT SOLUTION}

Einstein equation (3) can be solved by constructing characteristic curves. The result can be expressed as

$$
\begin{gathered}
-\left(t-t_{0}\right) \sqrt{2} E(r)+\sqrt{R(t, r)} \sqrt{E(r) R(t, r)+M(r)}-Q(r)=\frac{M(r)}{\sqrt{E(r)}} \ln \left[\frac{\sqrt{R(t, r)}+\sqrt{\frac{M(r)}{E(r)}+R(t, r)}}{\sqrt{R_{0}(r)}+\sqrt{\frac{M(r)}{E(r)}+R_{0}(r)}}\right] \\
Q(r) \equiv \sqrt{R_{0}(r)} \sqrt{E(r) R_{0}(r)+M(r)}
\end{gathered}
$$

for $E>0$ while for $E<0$, we have

$$
\begin{gathered}
F(r)-\left(t-t_{0}\right) \sqrt{2} E(r)=\frac{M(r)}{\sqrt{-E(r)}} \arcsin \left[\sqrt{\frac{-E(r)}{M(r)}} \sqrt{R(t, r)}\right]-\sqrt{R(t, r)} \sqrt{E(r) R(t, r)+M(r)} \\
F(r)=\frac{M(r)}{\sqrt{-E(r)}} \arcsin \left[\sqrt{\frac{-E(r)}{M(r)}} \sqrt{R_{0}(r)}\right]-\sqrt{R_{0}(r)} \sqrt{E(r) R_{0}(r)+M(r)} .
\end{gathered}
$$

Here, $R_{0}(r)$ is the initial condition specification for $R(t, r)$ : i.e. $R\left(t_{0}, r\right)=R_{0}(r)$. Note that the solution can be written a little more explicitly in the limit $|E M / R| \ll 1$ and $|E M / R| \gg 1$. For $|E M / R| \ll 1$, we find

$$
\begin{gathered}
R=\left(R_{0}^{3 / 2}+3 \sqrt{\frac{M(r)}{2}}\left(t-t_{0}\right)\right)^{2 / 3}\left(1+\frac{E(r)}{M(r)} \delta\right) \\
\delta=\frac{2 \cdot 2^{1 / 3} R_{0}^{3}+6 \cdot 2^{5 / 6} \sqrt{M} R_{0}^{3 / 2}\left(t-t_{0}\right)+9 \cdot 2^{1 / 3} M\left(t-t_{0}\right)^{2}}{5\left[2 R_{0}^{3 / 2}+3 \sqrt{2 M}\left(t-t_{0}\right)\right]^{4 / 3}}-\frac{2 R_{0}^{5 / 2}}{5\left[2 R_{0}^{3 / 2}+3 \sqrt{2 M}\left(t-t_{0}\right)\right]}
\end{gathered}
$$

For $E R / M \gg 1$, the leading order self-consistency requires

$$
R=R_{0}+\sqrt{2 E}\left(t-t_{0}\right)+\frac{M}{2 E} \ln \left[\frac{R}{R_{0}}\right]+\mathcal{O}\left(\frac{1}{E^{2}}\right) .
$$

Hence, we can approximate in the regime of interest

$$
R \approx \sqrt{2 E(r)}\left(t-t_{0}\right)+R_{0}(r)+\frac{M(r)}{2 E(r)} \ln \left(1+\frac{\sqrt{2 E(r)}}{R_{0}(r)}\left(t-t_{0}\right)\right) .
$$

